\title{
Liver Vessel Segmentation Algorithm Based on Fuzzy Enhance and Hessian Matrix
}

\author{
Yue-jing QIAN* \\ Collage of information and communications, Zhejiang Industry \&Trade Vocational \\ College, Wenzhou, Zhejiang, 325003, China \\ ${ }^{*}$ Corresponding author
}

Keywords: Hessian Matrix, Fuzzy Enhances, Blood Vessel Segmentation.

\begin{abstract}
This paper proposes a liver vessel segmentation algorithm based on fuzzy enhancement and Hessian matrix. First, a method with two steps based on the theory of fuzzy enhancement is proposed to enhance the liver image. The first enhancement step is to make the local changes in the image more obvious on the basis of fully retaining the image details. The second enhancement step is to enhance the edges by a sharpening parameter and a correction factor on the basis of the enhancement result gained by the first step, and so that the vessel areas in the image will be more obvious. Then the Hessian method is used to extract the vessel areas. Experiments show that the proposed method can segment the liver vessels accurately with a certain practicability and operability.
\end{abstract}

\section{Introduction}

Liver cancer refers to the tumor lesion occurred in the liver. Liver cancer has become the most important diseases to result in death. With the rapid development of computer application technology, the vessel automatic segmentation technology and the $3 \mathrm{D}$ reconstruction technology of vessels based on the CT scan image are becoming more and more important.

The prerequisite to get higher liver vessels 3D reconstruction accuracy is to segment the liver vessels accurately. The main method for liver vessels segmentation is first gaining the origin image by CT scanning the liver with vascular contrast agents, and then the vessels will be segmented and extracted based on some existing segmentation methods with the prior knowledge that the brightness of the vessels in CT scan image is more brighter than other tissues and organs in the liver.

The most commonly used segmentation are the methods based on boundaries, the methods based on region growing and the methods based on specific theories and tools. The segmentation methods based on boundaries mainly use some edge detection methods to segment and extract the vascular areas, such as Su Zhang et al. [1] proposed a pulmonary vessels segmentation method based on Canny operator and level set, Fetita et al.[2] proposed a fundus vessels edge detection method based on embedded confidence level. The segmentation methods based on the boundary are easily affected by noise with the results of discontinuous, and so, in order to improve the detection accuracy, the necessary human intervention is needed for the final results.

Region growing method first gets the pixels with similar nature together to constitute various areas, and then adds the other pixels to the corresponding areas according to the pre-defined standards from some seed pixels in these areas. Su Zhang et al. [1] proposed a method to reduce the scope of regional growth and to limit growth area boundary according to the gradient information between adjacent vessels images 
combined with mathematical morphology method. Fetita et al. [2] proposed a region growing method based on double rules of gradient amplitude change for vessels segmentation. Chen et al. [3] proposed a two-phase region growing method for liver vessels segmentation.

The segmentation methods based on specific theories and tools mainly use the prior structure of the liver vessels to construct a specific mathematical model to segment the vessels. Such as, Fetita et al. [2] put forward a senior morphological operator based on gray-scale (SDMC - connectioncost operator) to extract liver vascular structure. Chen et al. [3] split the intrahepatic vessels in a relatively small blood vessel by combining Graph-Cuts method with an iterative update region processing method. Goch et al. [4] proposed a vessels segmentation method by the gradient vector flow (GVF) and Frangis vessels segmentation method. Popkin et al. [5] put forward a new vessels segmentation algorithm based on the Hessian matrix.

Usually, image enhancement processing is needed before doing vessel segmentation. Image enhancement is one of the most commonly used in the field of image processing technology. According to the processing size of image region, image enhancement technologies can be divided into global enhancement and local enhancement. Global enhancement is to enhance the overall information of the image, however, this might easily lead to the local details missing, and so is not suitable for the segmentation of liver vessels processing. The traditional local enhancement methods include local histogram equalization, the noise removal method based on local statistical characteristics, adaptive histogram equalization with limited contrast], and so on. As the gray level of vessels in the liver area is relatively close the gray level of vessels surrounding tissues, the traditional local enhancement methods cannot obtain satisfactory enhancement effect. Tomohiro Takagi [6] proposed the mathematical method to construct fuzzy model, m. Wilscy [7] used the model for color image sharpening enhancing and gained good results. Based on this, this paper proposes a local enhancement algorithm based on fuzzy method for liver image enhancement processing.

\section{Local enhancement based on fuzzy method}

Local enhancement based on fuzzy method contains two steps: the first step is to calculated the absolute difference between the pixel in the neighborhood and the center pixel, then the membership function is used to build the weight, finally the enhancement result in this step can be gained by using a weighted summation method within the neighborhood pixels. The main purpose of the first step is to make the local change in the image more evident, and to prepare for the second edge enhancement step. The concrete realization processes of the first step are as follows:

Step1: Calculate the Euclidean distance between the current pixel and the pixels in the neighborhood: giving a neighborhood with the size of $3 \times 3$, for instance, and the current pixel is $(i, j)$, the gray value is $p_{i, j}$, then the distances between the pixels in the neighborhood and the center pixel are:

$$
d_{k, l}=\left|p_{i+k, j+l}-p_{i, j}\right|, k, l \in(-1,0,1) .
$$

Step2: Design the membership function, and then use it as the weight of each pixel 


$$
w_{k, j}=\left(\frac{k-d_{k . l}}{k}\right)^{2}, k=\max \left(d_{-1,-1}, \mathrm{~L}, d_{1,1}\right)
$$

Step3: Calculate the output result

$$
\text { out }_{i, j}=\sum_{k=-1, l=-1}^{k=1, l=1} w_{k, l} \mathbf{g} p_{k, l}
$$

The second step uses the image outputted from the first step as the image to be processed, and then enhances the edges in the image through a sharpening parameter $\lambda$ and a correction factor $\varepsilon$, and its implementation steps are as follows:

Step1: Calculate the gray level difference between the every pixel in the neighborhood and the center pixel

$$
\operatorname{dif}(k, l)=\operatorname{out}(k+i, l+j)-\operatorname{out}(i, j) .
$$

Step2: Calculate the correction factor $\varepsilon$

$$
\varepsilon(i, j)=\operatorname{Mean}(\text { dif }) \mathrm{g} \lambda \text {. }
$$

Step3: Calculate the final output results

$$
p(i, j)=\frac{\sum_{k=-1}^{+1} \sum_{l=-1}^{+1} \operatorname{out}(k+i, l+j)-\varepsilon(i, j)}{9}
$$

\section{Vascular enhancement based on the Hessian Matrix Eigenvalue}

Hessian method is a kind of method which uses the high order differential direction to extract image features. The direction and measures of the tubular structure are gained by the analysis of characteristics of $3 \mathrm{~d}$ gray-scale image Hessian matrix $\mathrm{H}$ :

$$
H=\left[\begin{array}{lll}
I_{x x} & I_{x y} & I_{x z} \\
I_{x y} & I_{y y} & I_{y z} \\
I_{x z} & I_{y z} & I_{z z}
\end{array}\right] .
$$

We can suppose that $\lambda_{1}, \lambda_{2}, \lambda_{3}$ are the three eigenvalues of $\mathrm{H}$ with corresponding eigenvectors $\lambda_{1}, \lambda_{2}, \lambda_{3}$ and their absolute values meet the conditions: $\left|\lambda_{1}\right| \leq\left|\lambda_{2}\right| \leq\left|\lambda_{3}\right|$. For a tubular structure, $v_{1}$ represents the direction with the minimum amount of gray level change, which is the direction of blood vessels. As tubular structures with different diameters only show the tube character under the scale of the specific scale, so it's necessary to calculate the vascular vector field under the framework of multi-scale to extract the blood vessels with different diameter. Multi-scale vector field is first to calculate the eigenvalue and eigenvector of a Hessian matrix under various scales, and then choose the largest measure as the final vector. We assume original image I, scale factor $\sigma$ with a range of [a1, a2], iteration step-size Step, and according to the papers, we can set the range of $\sigma$ as $[1,10]$, and set the iteration step-size as 2.The basic steps of blood vessels enhance are as follows:

Step1: Input image (gray image), and then get the pixel matrix I.

Step2: For each pixel $I_{x, y, z}$ in I, repeat the steps from Step3to Step10. 
Step3: Initialize the space scale $\sigma=\mathrm{a} 1$, and the enhance factor $z_{\max }=0$

Step4: If the space scale $\sigma$ meets the stop condition, do the step 9

Step5: Calculate the second order differential convolution of the element $I_{x, y, z}$ and Gaussian function.

Step6: Generate Hessian matrix $H$, and calculate the eigenvalues $\lambda_{1}, \lambda_{2}, \lambda_{3}$.

Step7: Calculate the output $z_{\max }$ of enhance filter.

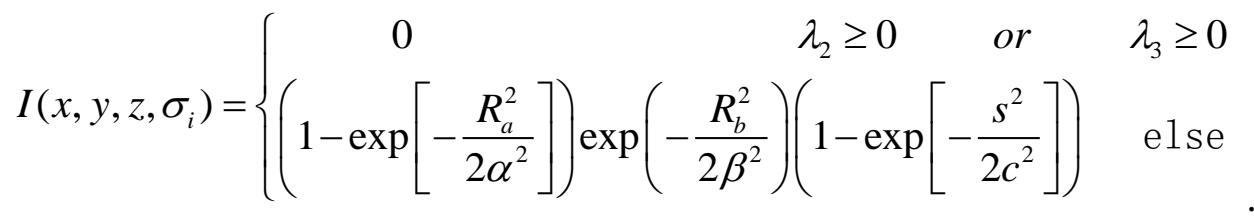

$$
\begin{aligned}
& R_{a}=\left|\frac{\lambda_{2}}{\lambda_{3}}\right|, \quad R_{b}=\frac{\left|\lambda_{1}\right|}{\sqrt{\left|\lambda_{2} \lambda_{3}\right|}}, \quad S=\sqrt{\sum_{j} \lambda_{j}^{2}} . \\
& z_{\max }=\max \left(Z_{\max }, I\left(V, \sigma_{i}\right)\right)
\end{aligned}
$$

According to the papers, we set the values of $\alpha, \beta$ as 0.5 , and set $\mathrm{C}$ as half of the biggest Hessian matrix norm.

Step8: Change the value of $\sigma$ with a specify step and return to step 4 to do iteration.

Step9: When the iteration ends, the enhance factor which is the biggest enhance filter output value $z_{\max }$ and the corresponding eigenvalues $\lambda_{1}, \lambda_{2}, \lambda_{3}$ and eigenvectors $v_{1}$, $v_{2}, v_{3}$ of Hessian matrix $\mathrm{H}$.

Step10: Judge whether the pixel is belonging to blood vessel based on the gained value of $z_{\max }$. That is the bigger value of $z_{\max }$, the more possible of the pixel being a part of blood vessel.

The characteristics of Hessian matrix eigenvalues are showing in the following Table 1.

\begin{tabular}{|c|c|c|c|c|c|}
\hline \multicolumn{2}{|c|}{$2 \mathrm{D}$} & \multicolumn{3}{|c|}{$3 \mathrm{D}$} & \multirow{2}{*}{ Direction of the pattern } \\
\hline$\lambda 1$ & $\lambda 2$ & $\lambda 1$ & $\lambda 2$ & $\lambda 3$ & \\
\hline \multirow[t]{3}{*}{$\mathrm{N}$} & $\mathrm{N}$ & $\mathrm{N}$ & $\mathrm{N}$ & $\mathrm{N}$ & noise image \\
\hline & & $\mathrm{L}$ & $\mathrm{L}$ & $\mathrm{H}-$ & The disk structure (bright) \\
\hline & & $\mathrm{L}$ & $\mathrm{L}$ & $\mathrm{H}+$ & The disk structure (dark) \\
\hline $\mathrm{L}$ & $\mathrm{H}-$ & $\mathrm{L}$ & $\mathrm{H}-$ & $\mathrm{H}-$ & Tubular structure(bright) \\
\hline $\mathrm{L}$ & $\mathrm{H}+$ & $\mathrm{L}$ & $\mathrm{H}+$ & $\mathrm{H}+$ & Tubular structure(dark) \\
\hline $\mathrm{H}-$ & $\mathrm{H}-$ & $\mathrm{H}-$ & $\mathrm{H}-$ & $\mathrm{H}-$ & Globular structure(bright) \\
\hline $\mathrm{H}+$ & $\mathrm{H}+$ & $\mathrm{H}+$ & $\mathrm{H}+$ & $\mathrm{H}+$ & Globular structure(dark) \\
\hline
\end{tabular}

Table 1. The Hessian matrix eigenvalues characteristics

\section{The Simulation Results}

Experimental hardware equipment is Intel dual-core processor $3.2 \mathrm{G}$ and $8 \mathrm{~GB}$ memory. The software and its version is matlab2014. Experiments are conducted to select one of the first image contrast, and then through the 3D imaging software to show the blood vessels segmentation effect. Figure 1.a is the original liver image, Figure 2.b is fuzzy 
enhancement liver images, after comparing two images, we can find that the liver vascular contrast with other regions in the enhanced image. Figure 2.a and Figure 2.b, respectively is the result of the linear transformation after the liver image, can see from the results, the enhanced image contains non vascular branches at the end of the less noise, more help to the rest of the blood vessel area on the basis of the Hessian method. Figure 3 is finally observed the different aspects of the blood vessels segmentation of 3 $\mathrm{D}$ visualization, by the diagram can be seen that the method can effectively segment the blood vessels in the liver.

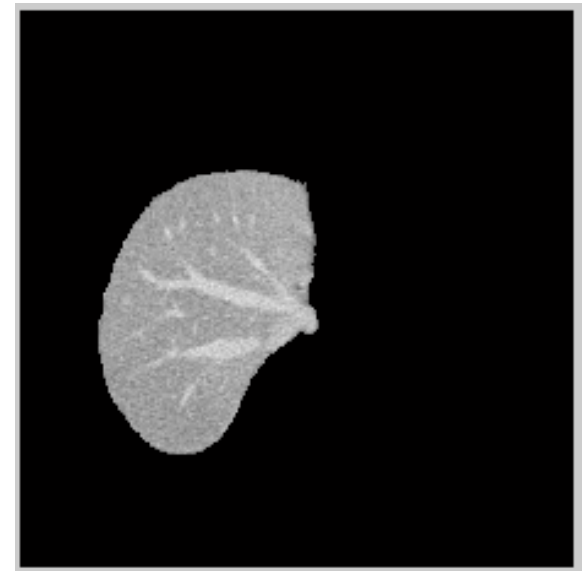

a

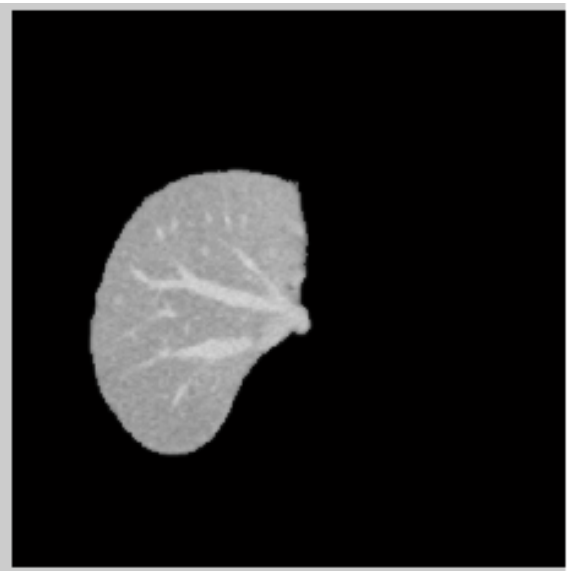

b

Figure 1. the original liver image and the fuzzy enhancement liver image

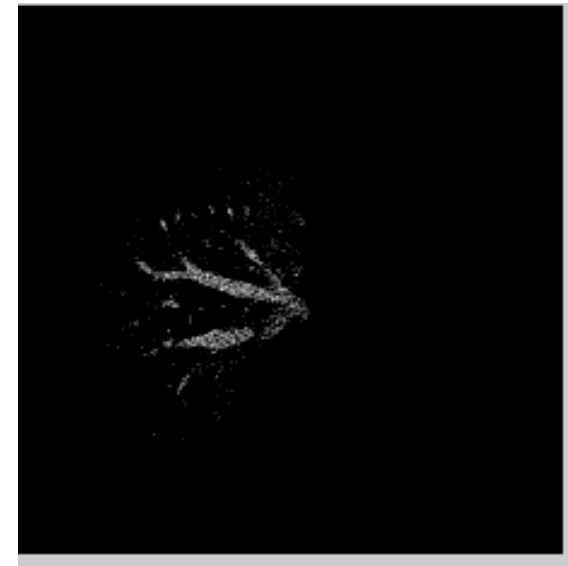

a

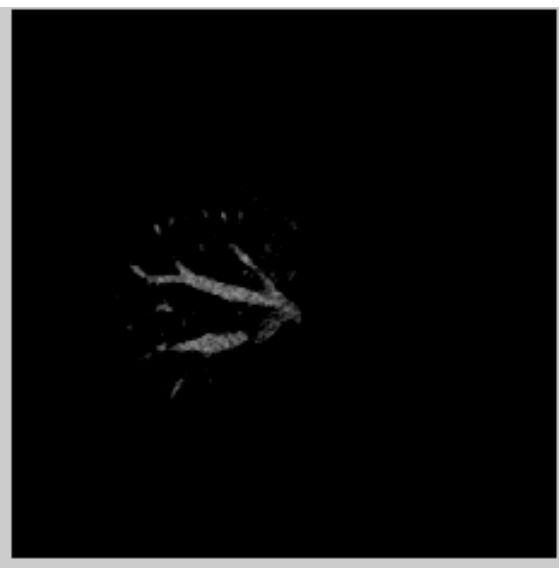

b

Figure. 2. The results of linear transformation in liver image

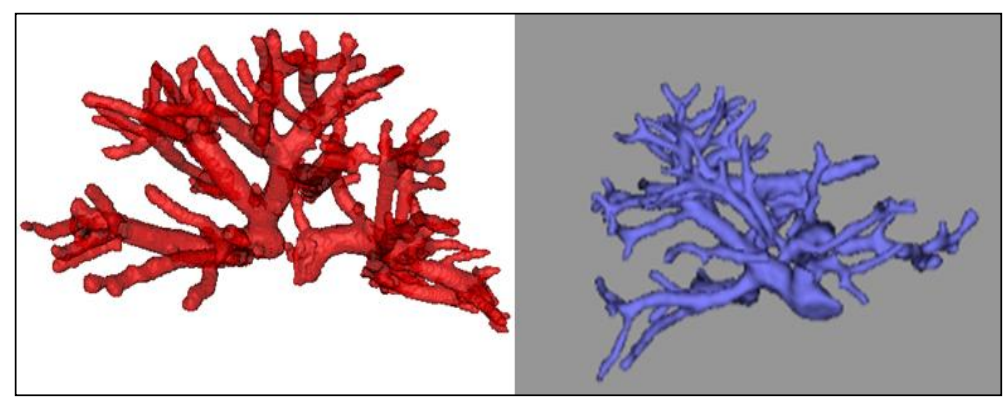

Figure. 3. Blood vessels with 3D visualization 


\section{Conclusion}

Local enhancement algorithm based on fuzzy theory is to enhance the overall image, to be able to vascular area, and other regional differences become more obvious. Based on strengthen the Hessian matrix eigenvalue is the enhancement of the tubular structure, the area can be used to extract blood vessels. First by the fuzzy method to enhance vascular area can further improve the late with Hessian method to extract the vascular area accuracy, the experimental results also verify the accuracy of the integrated algorithm, and less amount of calculation of the algorithm, implementation is simple and has a certain application value.

\section{Acknowledgement}

This research was financially supported by Wenzhou Science and Technology Project (S20150024) and Project funded by Zhejiang Provincial Department of Education (Y201432138).

\section{References}

[1] Su Zhang, Xuesong Lu, Yuanyuan Shen, et al. A new approach for automatic segmentation of LSCM blood vessel images of time sequence based on region growing [J]. IEEE/ICME International Conference on Complex Medical Engineering. 2007, 689-693.

[2] Fetita C, Lucidarme O.CT hepatic venography: 3D vascular segmentation for preoperative evaluation[C]//8th International Conference on Medical Image Computing and Computer-Assisted Intervention, 2005, 3750: 830-837.

[3] Chen Bichao, Sun Ying, Sim Heng Ong.Liver vessel segmentation using graph cuts with quick shift initialization[C]//Proceedings of the 15th International Conference on Biomedical Engineering, 2014, 43: 188-191.

[4] Goch C, Wang Xin, Meinzer H P, et al. Liver vessel segmentation using gradient vector flow[M]//Bildverarbeitung für die Medizin 2011.Berlin Heidelberg: Springer, 2011:104-108.

[5] Popkin T, Cavallaro A, Hands D. Accurate and efficient method for smoothly space-variant Gaussian blurring[J]. IEEE Transactions on Image Processing, 2010, 19(5): 1362-1370.

[6] Takagi, T., Sugeno, M.: Fuzzy identification of systems and its applications to modeling and control. IEEE Trans. Syst., Man, Cybern. SMC-15(1), 116-132 (1985)

[7] Wilscy M, Nair M S. A new method for sharpening color images using fuzzy approach[C]//International Conference Image Analysis and Recognition. Springer Berlin Heidelberg, 2008: 65-74. 